\title{
Las organizaciones de la sociedad civil en el contexto de la Cooperación Sur-Sur chilena: las potencialidades de una asociación estratégica para el desarrollo
}

\section{Catalina Pérez Gaete*}

\section{RESUMEN}

El presente artículo se centra en la relación entre las organizaciones de la sociedad civil (osc) y la Agencia Chilena de Cooperación Internacional para el Desarrollo (Agcid), en el marco de la evolución del sistema de cooperación nacional para el desarrollo, presentando los principales rasgos y características que aportan ventaja a las osc como agentes de cooperación, y enfatizando el rol complementario que cumplen en proyectos de desarrollo ejecutados por
Agcid. La finalidad de este artículo es relevar las potencialidades de las osc como agentes de cambio e integración para la css del país, en un escenario determinado por nuevos e inéditos desafíos para Agcid que requieren la consolidación de mecanismos innovadores de cooperación y de una estrategia multi-actores.

Palabras clave: Cooperación Sur-Sur; organizaciones de la sociedad civil; Agencia Chilena de Cooperación Internacional para el Desarrollo; Cooperación multiactor.

\footnotetext{
* Antropóloga social. Profesional de Apoyo en Generación de Contenidos para Dirección Ejecutiva, Agencia Chilena de Cooperación Internacional para el Desarrollo. Santiago (Chile). [cperez@agci.gob.cl]; [https://orcid.org/00000002-0048-5015].
}

Recibido: 13 de junio de 2019 / Modificado: 13 de agosto de 2019 / Aceptado: 14 de agosto de 2019

Para citar este artículo:

Pérez Gaete, C. (2020). Las organizaciones de la sociedad civil en el contexto de la Cooperación Sur-Sur chilena: las potencialidades de una asociación estratégica para el desarrollo. OASIS, 31, pp. 123-144

DoI: https://doi.org/10.18601/16577558.n31.08 


\section{Civil Society Organizations in the context of Chilean South-South Cooperation: the potential of a strategic partnership for develop- ment}

\section{ABSTRACT}

This article focuses on the relationship between Civil Society Organizations (csos) and the Chilean Agency for International Development Cooperation (Agcid), within the framework of the evolution of the national development cooperation system, presenting the main features and characteristics that provide an advantage to csos as cooperation agents, and emphasizing the complementary role they play in development projects executed by Agcid. The purpose of this article is to highlight the potential of csos as agents of change and integration for the country's ssc, in a scenario determined by new and unprecedented challenges for Agcid that require the consolidation of innovative cooperation mechanisms and a multi-actors strategy.

Key words: South-South Cooperation; Civil Society Organizations; Chilean Agency for International Development Cooperation; International Development; Multi-stakeholders Cooperation.

\section{INTRODUCCIÓN}

En los últimos años, la arquitectura de la Cooperación Internacional para el Desarrollo
(CID) ha experimentado profundos cambios en su composición. La irrupción de nuevos actores del desarrollo no-gubernamentales, ha delimitado una nueva cooperación más amplia e integradora. En este escenario, las Organizaciones de la Sociedad Civil (osc) han adquirido gradualmente un rol destacado en los procesos de integración y de cooperación.

En el caso de Chile, el país ha experimentado en los últimos años un cambio en su posición en el sistema internacional de cooperación, pasando de ser receptor neto de Ayuda Oficial al Desarrollo (AOD) desde principios de los años noventa, a ser un receptor disminuido, con una activa política de cooperación en términos cualitativos y cuantitativos, por medio de acciones de Cooperación Sur-Sur (css) y Cooperación Triangular. Además, el nuevo estatus del país como país graduado de renta alta ha promovido la búsqueda de mecanismos innovadores de cooperación en pos de incorporar más y nuevos recursos humanos, financieros y técnicos, mediante la incorporación de diversos actores del desarrollo a los esfuerzos de cooperación que realiza la Agencia Chilena de Cooperación Internacional para el Desarrollo (Agcid).

De manera particular, las osc poseen ventajas estratégicas frente a la cooperación que ejecutan las entidades públicas. No obstante, la nueva posición del país en el ámbito de la cooperación internacional ha implicado una serie de tensiones entre el Estado y la sociedad civil, que dificultan la articulación de un relato compartido entre ambos actores (Ramis, 2018).

El artículo busca destacar las potencialidades de las osc como agentes de cambio 
e integración para la css del país, en un escenario determinado por inéditos desafíos para Agcid, los que requieren la consolidación de mecanismos innovadores de cooperación multi-actorales.

En el segundo epígrafe, se analizan las causas y consecuencias de la (re)emergencia de la Cooperación Sur-Sur (css) en el sistema internacional de cooperación, caracterizando el rol de las osc en el contexto de la Agenda 2030.

Luego, en el tercer epígrafe se describe brevemente el recorrido histórico de las OsC en Chile, enfatizando el papel desempeñado por estas organizaciones durante la dictadura militar y, posteriormente, en el retorno de la democracia.

En el cuarto epígrafe se expone la evolución del sistema de cooperación internacional chileno, desde la creación de Agcid como institución encargada de canalizar la cooperación dirigida al país, hasta su nueva condición como país graduado, señalando los desafíos que esta posición implica para la cooperación nacional.

Después, en el quinto epígrafe se describen las potencialidades de las osc como actores del desarrollo en el marco de las alianzas estratégicas para el desarrollo, y a su vez, los retos que estas asociaciones deben afrontar para asegurar acciones de cooperación eficaces.
El sexto epígrafe presenta un conjunto de reflexiones finales que se derivan del análisis precedente. Finalmente, en el séptimo epígrafe se detalla la bibliografía consultada para la realización de este artículo.

Para cumplir con el objetivo señalado, el análisis de la información recopilada se efectuó mediante la técnica de análisis de contenido latente, utilizada en este caso para extraer el contenido simbólico de los discursos explícitos y/o implícitos presentes en los documentos seleccionados. Esto en el marco del paradigma hermenéutico, entendido como el estudio, análisis e interpretación de los textos, en el contexto del que forman parte (Rodríguez, et al., 1996; Hernández, 2014).

\section{EL (RE)SURGIMIENTO DE LA COOPERACIÓN SUR-SUR EN EL SISTEMA INTERNACIONAL DE COOPERACIÓN AL DESARROLLO}

Pese a que la css $^{1}$ existe desde hace décadas, su intensidad ha variado significativamente en los últimos años. Esto ha contribuido a aumentar el número de actores que participan en ella, y a diversificar enormemente los enfoques e instrumentos de CID.

En el caso de América Latina y el Caribe, en la primera década del siglo XxI se gestó un

\footnotetext{
Naciones Unidas define la css como "un amplio marco de colaboración entre paises del Sur en las áreas politica, económica, social, cultural, medioambiental y técnica que implica, al menos, a dos países en desarrollo y que puede hacerse en marcos bilaterales, regionales, subregionales o intrarregionales. En la CSS los paises comparten conocimientos, herramientas, experiencias y recursos para alcanzar metas de desarrollo por medio de esfuerzos concertados" (Naciones Unidas, en Ruiz, 2019). Por su parte, la Agcid considera a la css como aquella que "se realiza entre países de igual o menor desarrollo relativo bajo el principio de costos compartidos, resultado de experiencias compartidas y afinidades” (Ayllón, 2013).
} 
escenario idóneo para avanzar en la integración política, económica, social y cultural entre los países de la región, gestándose un escenario propicio para avanzar hacia una mayor autonomía frente a los centros tradicionales de poder. Esto a su vez motivó un renovado auge de la Css a nivel regional, la cual fue concebida como instrumento para lograr consensos, y alterar la naturaleza vertical, asimétrica y discrecional de la cooperación Norte-Sur. Esta modalidad de cooperación fue relevada por su potencial para fortalecer capacidades y transferir conocimientos, experiencias, recursos y soluciones técnicas en pos de un desarrollo mutuo entre países emergentes (Calvento y Rolandi, 2015; Santander, 2018; Ruiz, 2019).

Este apogeo de la css fue impulsado además por la crisis financiera que estalló el 2008 en los países del hemisferio norte. Ello detonó una importante reducción de los flujos de Ayuda Oficial al Desarrollo (AOD) hacia los países en desarrollo, mientras se consolidaba el empoderamiento que algunos habían logrado en las dinámicas económicas y políticas internacionales, ampliándose el abanico de las formas "tradicionales" de cooperación (HuitrónMorales, 2016; Ayllón, 2012).

A modo de síntesis, la revitalización de la css puede atribuirse a tres factores explicativos: 1) El relativo éxito económico de algunos países del Sur que alcanzaron considerables niveles de desarrollo socio-económico, y adquirieron nuevas tecnologías y competencias; 2) El rol que la cooperación internacional recibida tuvo en la adquisición de estas capacidades nacionales; 3) Y a la activación de un diálogo político entre países en desarrollo que comprendieron la necesidad de articularse en temas claves para su propio desarrollo (Ayllón, 2010).

El cambio estructural, que se orquestó en la gobernanza de la CID, promovió un trasvasije desde un sistema de cooperación unipolar, hacia uno multipolar, complejo y multidireccional con responsabilidades compartidas, aunque diferenciadas entre los distintos países. Al mismo tiempo, se produjo una difusión del poder hacia actores no-estatales, el cual se dispersó en estructuras y mercados que carecían de control público efectivo (Sanahuja, 2014; Santander, 2018).

\subsection{Las organizaciones de la sociedad civil como actores del desarrollo en el marco de la Cooperación Sur-Sur}

La CID ha experimentado en los últimos años un crecimiento cualitativo y cuantitativo exponencial que le ha permitido involucrar un mayor número de agentes e instituciones de distinta naturaleza, y con capacidades y orientaciones diversas (Ayllón, 2014).

De igual manera, los desafíos que plantea la "Agenda 2030 para el Desarrollo Sostenible ${ }^{2 "}$ demandaron una redefinición de la

2 La Agenda 2030 fue adoptada en el 2015 por 193 Estados como una hoja de ruta con el objetivo de afrontar los principales desafíos globales, con miras a un desarrollo sostenible e inclusivo. En este documento se plantearon 17 Objetivos de Desarrollo Sostenible (oDs) y 169 metas en los ámbitos económico, social y ambiental. 
gobernanza de la cooperación internacional, en pos de una cooperación multidimensional que incorporara nuevos marcos de asociación fortalecidos entre múltiples actores estatales y no-estatales, con potencial de movilizar conocimientos, capacidades técnicas y recursos (Cepal, 2018). Particularmente, las metas del oDs 17 "Revitalizar la Alianza Mundial para el Desarrollo Sostenible" se orientan al blindaje de los mecanismos de implementación de los oDs, incluyendo las alianzas multiactor en cuyo marco cada parte ponga a disposición sus ventajas para aportar al logro de objetivos comunes, reforzando a su vez las sinergias para lograr apalancar recursos (Surasky, 2017; Gutiérrez, et al., 2018).

Así, y pese a que la ciD es eminentemente pública respecto a su origen y la magnitud de los fondos que maneja, en los últimos años se ha incorporado, de forma gradual, a las empresas privadas, la Academia y la sociedad civil en general, componiendo todos ellos un sistema de redes de cooperación (Granguillhome, 2013).

No obstante, este sistema descrito no se encuentra institucionalizado en su totalidad, por lo que el concepto mismo de "sistema" resulta inexacto, en la medida que evoca la existencia de un esquema de cooperación articulado, cohesionado y coherente, lo que contrasta con la realidad, en la que el sistema de cooperación internacional para el desarrollo resultó de una lógica de agregación, donde no existió una entidad política central ni normas imperativas que determinaran inclusivamente la cantidad, formas de trabajo y criterios de asignación de la ayuda. Debe considerarse entonces que el término de "sistema" es aplicado en este contexto como unidad de análisis, haciendo alusión a una convención metodológica para distinguir sus elementos constitutivos -es decir, los actores que lo integran-, y las interacciones y dinámicas entre estos que dan orden y estructura al sistema internacional de cooperación internacional para el desarrollo (Ayllón, 2014).

Dentro de este sistema, la participación de las osc ha dotado a la css de una visión más amplia y representativa de los requerimientos reales de los países en desarrollo (Granguillhome, 2013), siendo estas entidades uno de sus pilares gracias a la creciente canalización de recursos de las agencias gubernamentales hacia estas organizaciones, además de su expertise acopiada en el campo del desarrollo, y su legitimidad social otorgada por sus vínculos con la ciudadanía, y las movilizaciones sociales nacionales y transnacionales (Ayllón, 2015b).

En el caso de América Latina y el Caribe, las osc han acompañado los procesos de democratización regionales, consolidándose como actores destacados en la elaboración e implementación de políticas públicas y en la promoción de los derechos humanos (DD.HH.) (Gutiérrez et al., 2018).

Tales objetivos fueron elaborados a través de consultas públicas, y luego de amplias negociaciones entre los países. En estas instancias deliberativas, la sociedad civil participó ampliamente, y de modo constructivo, en el proceso de configuración de esta Agenda (Rodríguez, 2017). 
En el 2000, la Declaración del Milenio reafirmó el compromiso para colaborar con las osc y otros actores para promover el desarrollo, lo que dio inicio a la discusión de las alianzas multiactor (González, 2018). Pese a esto, la sociedad civil no estuvo cuantitativamente presente en el desarrollo de la Declaración de París (2005) en parte porque las osc se resistieron a dedicar esfuerzos en una agenda que entendían estaba definida por los países donantes. No fue sino hasta el III Foro de Alto Nivel de Accra sobre la Eficacia de la Ayuda (2008) que las osc son reconocidas como actores plenos del desarrollo, siendo este papel reafirmado en el IV Foro de Alto Nivel de Busan (2011). Así, la sociedad civil fue incorporada como miembro pleno de los órganos de seguimiento y negociación de la agenda de desarrollo, involucrándose gradualmente en las redes internacionales que se configuraron en estos espacios (Alemany y Freres, 2018).

Cabe destacar que, a diferencia del reconocimiento que las osc han alcanzado en los países de la Organización para la Cooperación y el Desarrollo Económicos (Ocde), no ha ocurrido lo mismo en la css, pese a que estas organizaciones han contribuido a asegurar su boom en la región. Así, su participación en este ámbito es uno de los aspectos menos explorados en los estudios de CiD. Ello porque la mayoría de las investigaciones establecen como unidad de análisis a los Estados nacionales, existiendo pocas referencias sobre la participación de los actores no-estatales en la css latinoamericana. Se trata así de una agenda de investigación pendiente que podría eventualmente guiar a una segunda generación de estudios sobre la css en la región (Calvento y Rolandi, 2015; Ayllón, 2015a; Soto y Viveros, 2016).

\section{LAS ORGANIZACIONES DE LA SOCIEDAD CIVIL COMO ACTORES DE LA COOPERACIÓN INTERNACIONAL CHILENA}

\subsection{Las organizaciones no gubernamentales como receptores de flujos de ayuda externa en dictadura}

De modo general, las osc ${ }^{3}$ surgen a partir de la motivación de grupos de individuos que buscan proveer de bienes y servicios a determinados sectores de la población que requieren asistencia, o bien buscan movilizar el interés público en dirección a temáticas y demandas sociales específicas. Desde sus inicios, estas organizaciones fueron entendidas como entes sociales distintos del Estado y el mercado con características propias, desplazándose en espacios acotados de acción y adaptándose de modo dinámico a los distintos escenarios políticos, económicos y sociales de los países (Díaz de Valdés, 2016; Soto y Barrientos, 2016).

En el caso de Chile, históricamente ha existido una tradicional presencia de las osC en el ámbito de la satisfacción de necesidades sociales, la implementación de políticas socia-

\footnotetext{
3 En el contexto de este artículo, entenderemos las osc como organizaciones conformadas por personas que se asocian en grupos estructurados, con base en normas, intereses y objetivos particulares, para dar respuesta a necesidades sociales, grupales y colectivas (Acotto, en González, 2018).
} 
les, y en el desarrollo de comunidades locales y de pobladores en sectores urbanos precarios (Soto y Barrientos, 2016).

Durante la dictadura militar (1973-1990) proliferaron en Chile diversas organizaciones de base opositoras a este régimen, movilizadas por la voluntad de reivindicar los DD.HH., y apoyar a los sectores sociales severamente afectados por las políticas económicas del régimen militar, operando en un contexto de supresión de derechos sociales, de violenta represión política y de fuerte reducción del gasto social (Gruninger, 2003; Delamaza, 2009).

Estas instituciones -denominadas "organizaciones no gubernamentales" (ONG)- se articularon mayoritariamente en los años ochenta, gracias al respaldo financiero que gobiernos y organismos económicos multilaterales canalizaron hacia espacios alternativos de la sociedad civil. De este modo, uno de los principales factores que incidió en la expansión de las ONG fue el aumento de la oferta de cooperación internacional hacia Chile, promovida por el rechazo internacional que suscitó el terrorismo de Estado, y también por las paupérrimas cifras de pobreza resultantes del modelo de desarrollo adoptado por la dictadura chilena (Fernández, 2018).

\subsection{El rol subsidiario de las ong en la transición democrática de Chile}

El retorno de la democracia en 1990 originó una activa reinserción de Chile al ámbito de la cooperación internacional, percibiendo a principios de la década de los noventa la ayuda de otros países que buscaron apoyar el desarrollo nacional. Este proceso suscitó un traspaso de la cooperación internacional hacia el Estado, el que fue acompañado paralelamente por una transferencia de capacidades técnicas y profesionales desde las ONG hacia el Estado chileno (Egaña, 1991; Delamaza, 2009).

De manera abrupta, las ONG se vieron privadas de fuentes de financiamiento, lo que terminó por debilitarlas, y las presionó para buscar nuevos modos de asociación con el sector público. Paulatinamente, estas organizaciones se articularon de un modo subordinado al Estado como prestadoras de servicios externalizados, función que les permitió asegurar su sostenibilidad y su permanencia en el tiempo. Sin embargo, sus saberes y conocimientos fueron excluidos de los procesos de transición democrática (Soto y Barrientos, 2016; Díaz de Valdés, 2016).

La relación que se dio en términos de consultoría entre las ONG y el Estado puso en jaque el carácter no lucrativo de estas organizaciones, y las obligó a competir por asignaciones de fondos públicos para compensar la pérdida de recursos que provenían de la cooperación. Este escenario condujo inevitablemente a tensionar y fracturar las redes erigidas entre las ONG durante la dictadura, lo que tuvo como resultado su atomización (Delamaza, 2009; Fernández, 2018).

\section{4.- CHILE COMO OFERENTE DE COOPERACIÓN SUR-SUR}

\section{1.-La creación de la Agencia Chilena de Cooperación Internacional}

Para canalizar la ayuda que el gobierno comenzó a recibir a partir del retorno de la democra- 
cia, fue creada por la Ley Nº 18.989 la Agencia Chilena de Cooperación Internacional (Agci $\left.{ }^{4}\right)$ en 1990, como organización instrumental del Estado, responsable de gestionar la cooperación internacional de carácter concesional y de articular el sistema nacional de CID. Este sistema se caracteriza por su alta fragmentación, puesto que varias instituciones y ministerios realizan acciones de cooperación a través de sus áreas o direcciones de asuntos internacionales. Si bien Agcid se configura como el actor central de este sistema, la evolución que ha tenido el rol de la CID en nuestro país ha permitido a distintos actores (regiones, empresas, sociedad civil, entre otros) participar en ella, generando la necesidad de mejorar el conocimiento mutuo y las sinergias entre estos (Lazo, 2012; Agcid, 2015; Amexcid, 2019).

Luego del retorno de la democracia, las ONG buscaron instaurar un diálogo con la Agcid, cifrando sus expectativas en que la Agencia asignaría recursos para otorgar soporte al fortalecimiento de las osc de acción pública social. Empero, la Agcid optó por no participar en el establecimiento de esta propuesta, dando prioridad a iniciativas de naturaleza política que fueran en beneficio de instituciones que respaldaban al nuevo gobierno (Fernández, 2018).

\section{2.-El modelo de Cooperación Sur-Sur de Chile}

A mediados de los años noventa, Chile experimentó un prometedor escenario económico, con tasas de crecimiento cercanas al 6\% anual, y una estabilidad política que le permitió proyectarse más allá de sus fronteras (López, 2015).

En 1993, Agcid creó el Programa de Cooperación Horizontal, con el objetivo de compartir las capacidades, los conocimientos y las experiencias que Chile acumuló en materia de políticas públicas durante los primeros años de democracia, con países de menor o similar desarrollo, otorgando prioridad en sus acciones a los países de América Latina y el Caribe (Sánchez, 2010).

Desde un comienzo, los gobiernos democráticos optaron por robustecer la inserción internacional del país por medio de una amplia liberalización económica, materializada principalmente mediante acuerdos y tratados de libre comercio, y la atracción de inversión extranjera. Para aportar a cumplir este objetivo, la cooperación internacional fue empleada para afianzar el modelo de inserción económica externa del país, cumpliendo una función instrumental en pos de crear "buena voluntad" para las empresas chilenas en los mercados

4 En el 2018, la Agencia cambia su nombre, desde Agencia de Cooperación Internacional de Chile (Agci) al de Agencia Chilena de Cooperación Internacional para el Desarrollo (Agcid). Para efectos de este artículo, utilizaremos el término Agcid. 
regionales y globales (Santander, 2018; Giacalone, 2016). Así, el modelo de cid que surgió combinó la solidaridad con la intención de forjar buenas relaciones vecinales y regionales, distinguiéndose de las osc, cuyo discurso se sustentaba en las carencias estructurales del país (Ramis, 2018).

En la medida en que Chile fue consolidando una doble dimensión en materia de CID -vale decir, como receptor y socio de cooperación- la gestión de Agcid dio un salto cuantitativo y cualitativo ${ }^{5}$, impulsado en parte por el compromiso que el país asumió de cooperar con otros. Al mismo tiempo, el mayor desarrollo relativo mostrado por el país en relación con el entorno regional promovió el establecimiento de programas de becas y esquemas de cooperación técnicas, dentro de los cuales el modelo de css chileno fue adquiriendo forma. Todo lo anterior confluyó en un cambio de paradigma en la cid chilena ${ }^{6}$, en cuyo marco se establecieron vínculos moderados de carácter institucional con la sociedad civil (Agci, 2010; Santander, 2018).

El modelo de css de Chile se sustentó en la auto-percepción del país como actor de cooperación con capacidades diferenciadas del entorno regional, afectando acciones de cooperación en áreas específicas en las cuales Chile posee expertise positivamente valorada por sus contrapartes. Esto a su vez, ha configurado un patrón de cooperación con escasos sesgos ideológicos al momento de elegir a los países destinatarios de CID chilena (Agcid, 2018; Santander, 2018).

No obstante, el Estado chileno ha dispuesto de recursos financieros más limitados en css que otros países en desarrollo, perfilándose como un socio germinal en cuanto a capital financiero. De esta manera, la inclinación de Agcid por gestionar acciones de cooperación puntuales de alta dispersión geográfica se vincularía a los acotados montos de los que ha dispuesto, usufructuando así de la ventajosa relación coste/beneficio, que posee la CID. La cooperación fue entonces instrumentalizada por el Estado como "poder blando" de la política exterior, en pos de percibir retornos en forma de prestigio e influencia al país (Nye, en Ayllón, 2012; Santander, 2018).

De igual manera, la cooperación triangular ha generado beneficios relativos a la gestión del conocimiento, permitiendo incrementar la cooperación que el país otorga. La triangulación opera como un mecanismo que facilita el diálogo entre las demandas de cooperación de la región, y la oferta de los donantes tradicionales, posibilitando a Chile consolidar una posición como socio confiable en el ámbito de la css (Guerra, 2014).

\footnotetext{
A partir del año 2005, y según Ley 19.999, Agcid empieza a relacionarse con la Presidencia de la República a través del Ministerio de Relaciones Exteriores, lo que convierte a la cooperación en un instrumento para reforzar los propósitos y objetivos de la política exterior de Chile (Guerra, 2014).

6 Durante el gobierno de M. Bachelet (2006-2010) se hizo explícita la adopción de la css, resultado de la revisión de la arquitectura y los mecanismos del sistema de la cid (Van Klaveren, 2011).
} 


\subsection{La graduación de Chile como país de renta alta}

Agcid ha transitado en los últimos años desde una posición como receptor neto de Ayuda Oficial al Desarrollo (AOD) en los albores de la década noventa, a desempeñar un rol dual en el ámbito de la css, el cual implica poner a disposición de otros capacidades, recursos, experiencias y buenas prácticas, considerando a su vez las contribuciones que otros países puedan aportar al país en áreas en las que Chile aún requiere de cooperación, como son protección del medio ambiente, provisión de bienes públicos globales, energías renovables, cohesión social, innovación e infraestructura económica (Santander, 2018; Ramis, 2018).

Pese a las necesidades de cooperación que el país presenta aún, en el 2013, el Comité de Ayuda para el Desarrollo (CAD), perteneciente a la Ocde, revisó la lista de países elegibles para la AOD, determinando que Chile se graduaría en 2017 como país de renta alta si continuaba superando el umbral de renta per cápita fijada por el Banco Mundial (aproximadamente 12.700 dólares). Esto finalmente ocurrió, y constituye para el país una situación irreversible. Una de las principales consecuencias de este suceso es el cambio de estatus de Chile en el sistema internacional de cooperación para el desarrollo, gracias al cual deja de ser considerado como un país que requiere de cooperación.
Esto ha generado importantes restricciones de cooperación para el país, como son la reducción de oportunidades de formación de capital humano, la imposibilidad de continuar percibiendo asistencia técnica en áreas aún deficitarias para el desarrollo nacional, y el impedimento de acceder a créditos en condiciones ventajosas (blandos) otorgados por los bancos de los países donantes del CAD (Ampuero, 2018; Hernández y Lebret, 2019). Por lo demás, Chile se encuentra alejado de los promedios de cooperación de los países de este Comité, considerando el porcentaje del PIB que el país destina a este ítem ${ }^{7}$.

Por ello, desde el 2016, Chile viene impulsando al interior del CAD/Ocde, una estrategia de mitigación cuyo foco son los desafíos particulares que los países de renta media alta enfrentan para completar sus procesos de desarrollo, como es la desigualdad entre y al interior de los países. El país ha argumentado, en diversos espacios multilaterales, que el uso del ingreso per cápita como único criterio para graduar constituye una forma de exclusión que no da cuenta del carácter multidimensional del desarrollo, y que invisibiliza las brechas estructurales que mantienen aún los países en desarrollo (Agcid, 2018; Hernández y Lebret, 2019). Chile ha buscado así seguir percibiendo cooperación de las fuentes tradicionales, y al mismo tiempo, fortalecer iniciativas de triangulación y mecanismos innovadores de

7 Mientras en el 2017 la AOD total proveniente de los miembros del CAD equivalió al $0.31 \%$ de su Producto Nacional Bruto, la Ley de Presupuesto del 2017 otorgó a Agci una cifra cercana a los 8 millones de dólares, lo que equivale aproximadamente a un 0,002\% del PIB de Chile ese año. Información disponible en https://datos.bancomundial.org/ 
asociación para movilizar más y nuevos recursos (Agcid, 2018).

\subsection{La adopción de una Cooperación Multiactores para el desarrollo}

En los últimos años, Chile ha consolidado su posición regional y global a través de mecanismos de financiamiento que aportan al ods 17, es decir, a las alianzas para el desarrollo. Esto ha permitido diversificar la cooperación chilena, incorporando la participación de la sociedad civil en pos de avanzar en inclusión y sostenibilidad (Agcid, 2019).

Para este propósito, Agcid ha impulsado iniciativas de cooperación que involucran a múltiples actores del desarrollo, estimulando la conformación de alianzas multiactores, entendidas como esquemas de cooperación voluntaria y de carácter horizontal entre el sector público y otros actores, con el objeto de desarrollar iniciativas que colaboren en la implementación de los ods (Apci, 2016; Agcid, 2018). Este proceso ha sido motivado por la nueva posición de Chile en el sistema de cooperación internacional, y la disminución progresiva de la AOD destinada al país (véase figura 1).

De este modo, Agcid ha puesto en marcha iniciativas de cooperación inclusivas, destacando la creación de una Mesa Multi Actores en el 2017. Esta Mesa constituye un espacio para trabajar y dialogar con actores de la sociedad civil -principalmente sector privado, funda-

Figura 1

Ayuda oficial neta para el desarrollo recibidas por Chile 1985-2017 (us\$ a precios actuales)

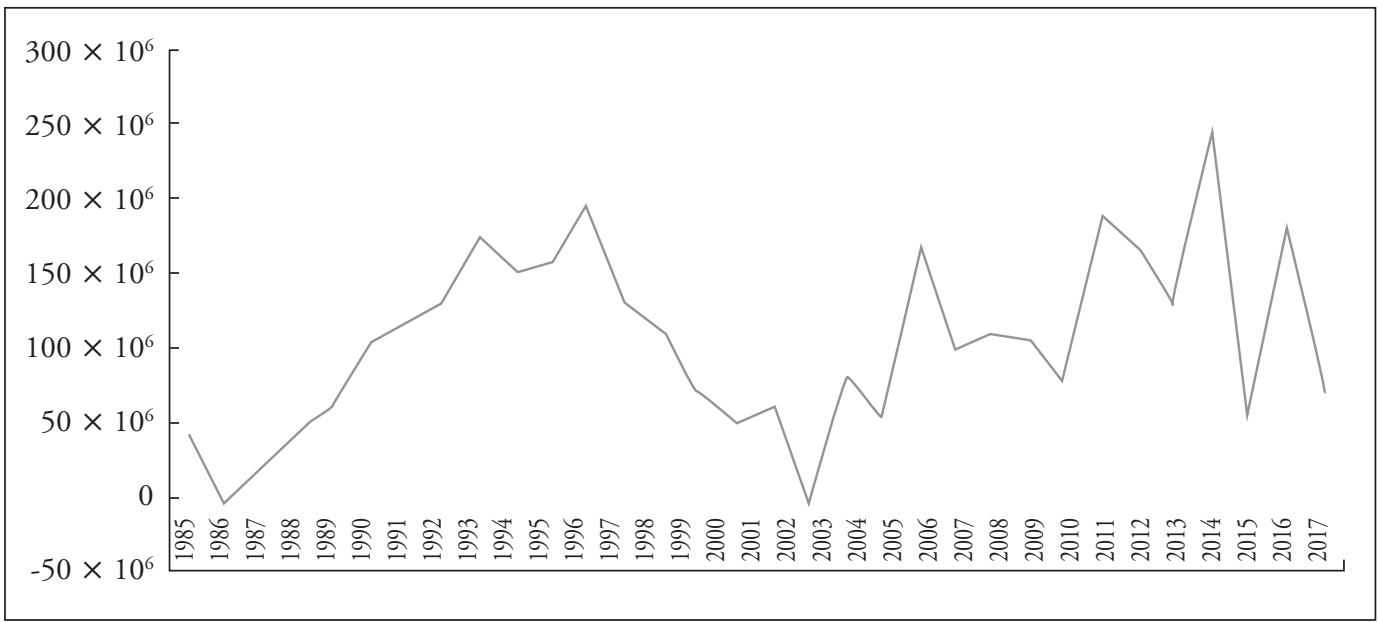

Fuente: Elaboración propia a partir de Banco Mundial. Información disponible en https://datos.bancomundial.org/ indicador/DT.ODA.ALLD.CD?locations=CL\&view=chart 
ciones y universidades- con el propósito de fomentar una CID plural e integradora (Agcid, 2018).

De igual manera, la conformación del Consejo de la Sociedad Civil ${ }^{8}$ (Cosoc), integrado por representantes de la sociedad civil vinculados al quehacer de Agcid, sobresale como un valioso mecanismo de participación ciudadana. Este Consejo ${ }^{9}$ posee carácter consultativo y autónomo, y su principal función es actuar como acompañante en los procesos de toma de decisiones en políticas públicas. No obstante, se debe señalar que el rol de este Consejo aún es incipiente (Ramis, 2018).

Resulta importante destacar la elaboración de un primer documento de Política de Cooperación Internacional para el Desarrollo de Chile en el 2015, que adopta una mirada más compleja y matizada del desarrollo. En el marco de esta Política se aprobó la primera Estrategia de cooperación internacional del país para el período 2015-2018. En esta hoja de ruta, se explicita la búsqueda de Agcid por abrir nuevos canales de trabajo con múltiples actores, reconociendo el rol de la sociedad civil y sus capacidades instaladas en el ámbito de la CID, así como los conocimientos que estas entidades han acopiado en temáticas relevantes para la css chilena (Agci, 2015).

\section{5.- LAS ORGANIZACIONES DE LA SOCIEDAD CIVIL COMO ACTORES RELEVANTES EN LAS ALIANZAS ESTRATÉGICAS PARA EL DESARROLLO}

\section{1.-Caracterización de las Organizaciones de la Sociedad Civil en Chile}

En Chile existen más de 240 mil osc oficialmente inscritas, de las cuales cerca de un $48 \%$ se registraron entre 2006 y 2015. La reciente conformación de parte importante de estas organizaciones puede adjudicarse a la promulgación de la Ley 20.500 (2011), que agilizó los procesos de inscripción y redujo los costos asociados a la constitución de las osc. De igual manera, este fenómeno puede explicarse también por un estímulo proveniente de la misma sociedad civil moderna por querer establecerse como organizaciones formales (Irarrázaval et al., 2016).

En cuanto a su vocación, alrededor de un $32 \%$ se dedica a actividades vinculadas a desarrollo social y vivienda, mientras que aproximadamente un 31\% se ocupan en labores de cultura y recreación. Más atrás, en tercer y cuarto lugar, se posicionan aquellas osc que desarrollan iniciativas de investigación y educación, y aquellas relacionadas a asociaciones

\footnotetext{
8 El Cosoc de Agcid está integrado por: la Asociación Chilena de Voluntariados, la Universidad Católica del Maule, la Universidad Católica del Norte, la Fundación Ciudadano Inteligente, Asociación Chilena de ONG ACCiON, Corporación Asociación Chilena Pro Naciones Unidas, Fundación Un Techo para Chile, Sindicato Independiente de Trabajadoras Sexuales Transgéneras Amanda Jofré, y Fundación Instituto de Ecología Política (Acta Sesión Ordinaria N 12, 25 de marzo de 2019). Información disponible en https://www.agci.cl/documents/acta_12_CSC_AGCI_2019. pdf

9 Información disponible en https://www.agci.cl/index.php/consejo-de-la-sociedad-civil
} 
gremiales, asociaciones profesionales y sindicatos respectivamente. Finalmente, las osc con menor presencia en Chile corresponden a las organizaciones internacionales, $y$ las de intermediarios filantrópicos y promoción de voluntariado (Irarrázaval et al., 2016).

Es importante mencionar que, si bien no todas las osc se encuentran vinculadas a la CID, la mayoría se han integrado en espacios de debate regionales y globales sobre temas internacionales significativos (Moreno, 2019).

\subsection{Las ventajas de las organizaciones de la sociedad civil como actores del desarrollo}

La nueva posición de Chile en el sistema de CID ha implicado una serie de tensiones entre el Estado y la sociedad civil, agravadas por los efectos negativos de las políticas neoliberales acumulados por cerca de cuatro décadas en el país. Esto propició un estado de insatisfacción social y política generalizada, promovida por el declive del crecimiento global, y que derivó en un cuestionamiento de la legitimidad de las instituciones democráticas y de los actores políticos, que tuvo como protagonistas a las osc (Sánchez et al., 2012; Fernández, 2018).

Esta crisis de confianza hacia las instituciones en el país alentó un favorable auge de las osc, favorecido por sus potencialidades para complementar la labor del Estado en materia social. De este modo, entre ambos sectores se ha forjado una relación de interdependencia caracterizada por una progresiva demanda de recursos de parte de las Osc, y al mismo tiempo, una búsqueda de estas por concitar mayor apoyo por parte del ejecutivo a su existencia y su labor (Díaz de Valdés, 2016).

En el ámbito de la CID, estas organizaciones se han posicionado como inestima-

\section{Organizaciones de la sociedad civil según actividad}

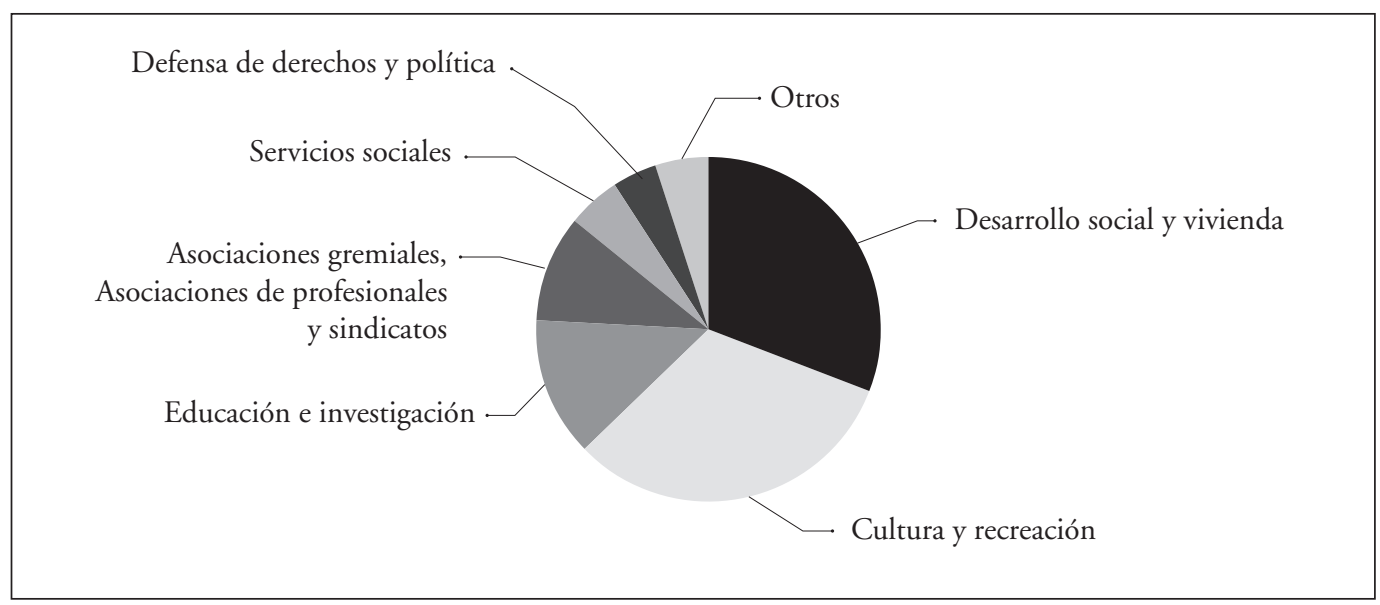

Fuente: Elaboración propia a partir de información disponible en Irarrázaval et al., 2016 
bles fuentes de conocimientos, recursos y capacidades, desempeñando un rol doble $-\mathrm{y}$ complementario- en la gobernanza de la CID (Granguillhome, 2013; Apci, 2016). Por un lado, son co-partícipes en las discusiones sobre la eficacia de la CID, aportando con una perspectiva social en el seno de estos debates. Por otro lado, poseen un destacado papel como ejecutoras de acciones de CID, y ostentan renombrada experiencia en recaudación de fondos. Así, en años recientes, muchas de estas organizaciones han conseguido forjar valiosas redes en el extranjero que favorecen el intercambio de conocimientos entre sus miembros, promueven una mayor eficacia y eficiencia de la CID, aportando de igual manera al fortalecimiento de la democracia en muchos países (Weber, 2011).

En el 2018, 23 organizaciones -específicamente ONG- de la sociedad civil ${ }^{10}$ participaron en el quehacer de Agcid, en proyectos bilaterales y triangulares (Agcid, 2019). No obstante, las osc chilenas poseen aún una baja participación en acciones de cooperación gestionadas por Agcid. Este es un pendiente que debe ser subsanado, considerando las ventajas que estas organizaciones poseen para efectuar acciones de desarrollo, entre las cuales podemos destacar (Sánchez et al., 2012; Zamora y Molina, 2016; Díaz de Valdés, 2016; Moreno, 2019):

- Presentan una amplia diversidad y heterogeneidad en sus capacidades, funciones y en sus recursos financieros y humanos. Asimismo, gozan de gran flexibilidad para planificar, y de importantes competencias para efectuar diagnósticos tempranos.

- Representan un modelo de economía solidaria y una forma de expresión de la ciudadanía, y de sus intereses. Además, sostienen mejores canales de comunicación con la ciudadanía y los medios de comunicación, manteniendo gran cercanía con los problemas locales.

- Pueden canalizar y gestionar mayor participación civil, favoreciendo el robustecimiento de capacidades en las comunidades de las que son parte, y el desarrollo de la diversidad, la creatividad, la tolerancia y el sentido de pertenencia de las y los ciudadanos.

- Dan sustento a la democracia en un marco de relaciones de confianza y reciprocidad.

- Desarrollan bienes públicos globales como educación, justicia, DD.HH. e igualdad, entre otros, aportando a la cohesión e inclusión social mediante la reducción de las asimetrías al interior de las sociedades.

- Fortalecen la probidad ciudadana por medio de la autorregulación colectiva.

- Cumplen un rol fiscalizador sobre el uso de los recursos que emanan de la css, instando a aumentar la transparencia de los mismos, y aportando a la rendición de cuentas de los gobiernos.

10 Además, Agcid trabajó con 44 instituciones académicas (Universidades y Centros de Estudio), y 9 entidades del sector privado (Agcid, 2019). 
- $\quad$ Algunas osc que han destacado por sus altos niveles de profesionalización, y por su facultad para acceder a fuentes económicas de la empresa privada.

- Disponen en algunos casos de más y mejor personal especializado y conocimientos en terreno para ejecutar proyectos que los Estados.

- Tienen una visión multigradual de la CID, y coinciden con propuestas de la ciudadanía regional en cuanto apelan a construir una regionalización más allá de la revisión de acuerdos comerciales.

Cabe destacar que los aprendizajes emanados de la experiencia de las osc para movilizar voluntariado constituyen un potencial de amplio valor para Agcid, considerando las nuevas responsabilidades que la Ley Nº 21.080 de Modernización de Cancillería confiere a la Agencia en temas de ayuda humanitaria (Ramis, 2018).

En resumen, la consolidación de una perspectiva compartida entre sector público y sociedad civil permitiría ampliar y consolidar el rol de Chile como actor para el desarrollo en el marco de la cCs. Esto porque las osc ocupan un lugar central en el entramado de la sociedad actual, sustentado en sus amplias virtudes como su flexibilidad, rapidez, economía de recursos escasos, cercanía a problemas locales y expertise acumulada (Díaz de Valdés, 2016).

\subsection{La apertura de nuevos espacios de colaboración entre Estado y sociedad civil: el caso del Fondo Chile contra el Hambre y la Pobreza}

En los últimos años, los países de ALC han adoptado mecanismos de financiamiento innovadores complementarios a las formas tradicionales de cooperación, en sintonía con los cambios en el panorama del financiamiento para el desarrollo (Cepal, 2015).

A comienzos del nuevo siglo, las osc locales y globales comienzan a abandonar progresivamente su rol instrumental, adquiriendo mayor poder y autonomía en procesos de reivindicación y conquista de derechos sociales y medioambientales, aportando asimismo a construir una democracia más participativa. Diversos organismos internacionales y agencias de cooperación al desarrollo, impulsados por la identificación de problemáticas estructurales del desarrollo, comenzaron a modelar políticas y programas destinados al fortalecimiento de la sociedad civil, multiplicándose de esta forma las fuentes de financiamiento internacional para las OsC (Ballesteros, en MSGG, 2016).

En este contexto, destacan los fondos de cooperación ${ }^{11}$ como mecanismos innovadores para el desarrollo, de entre los cuales, el Fondo Chile contra el Hambre y la Pobreza (FCHP) ha destacado como un hito respecto al forta-

11 Otros fondos implementados por Chile con éxito son el Fondo Mixto Chile-España y el Fondo Chile-México (Agcid, 2015).

OASIS, ISSN: 1657-7558, E-ISSN: 2346-2132, N³1, Enero - Junio de 2020, pp. 123-144 
lecimiento de la participación de las osc en el marco de la CID regional y global.

Chile participa desde el 2006 en esta iniciativa internacional, que buscó hacer correcciones en la globalización financiera y suministrar financiamiento adicional para combatir la pobreza, fomentando una globalización con rostro humano. En este contexto, Chile fue el primer país en introducir tasas de embarque de us\$ 2 dólares destinadas a ampliar la cobertura de acceso a diagnósticos y tratamientos para el $\mathrm{vIH} /$ Sida, la malaria y la tuberculosis (FfrenchDavis, 2016).

Posteriormente, el gobierno canalizó parte de estos fondos para financiar proyectos de cooperación internacional, en colaboración con el Programa de Naciones Unidas para el Desarrollo (Pnud), creándose el FCHP. Este instrumento de cooperación fue dotado de respaldo legal que le otorga suficiente estabilidad y solidez temporal para establecer una línea de acción orientada a colaborar con las OsC (Ffrench-Davis, 2016; Agcid-Pnud, 2019).

Con el FCHP se logra diversificar el rol de estas organizaciones en el marco de la css del país, aportando mayor eficacia a los vínculos colaborativos de Agcid con los países vecinos, y expandiendo las posibilidades para todos los actores involucrados de beneficiarse con los conocimientos y la expertise provenientes de la totalidad de los agentes implicados. De igual manera, el FCHP potencia a las osc de menor envergadura y menor peso económico al permitirles postular en alianzas (Ramis, 2018; Moreno, 2019).

En el marco de este Fondo, se han financiado más de 75 proyectos, por un monto cercano a los usD \$12 millones de dólares en Amé- rica Latina y el Caribe, África y Asia Pacífico. De estas iniciativas, cerca de un 60\% han sido ejecutadas por osc chilenas. En consideración a la segunda fase del FCHP (2015-2020), existen en el 2019 cuatro proyectos de cooperación en los que estas entidades participan como socias de cooperación (Agcid-Pnud, 2019).

Existen ciertas osc que han conseguido importantes avances en la construcción de redes internacionales, y que fueron configurando un enfoque de cooperación basado en la duplicación de experiencias de voluntariado y la participación ciudadana, logrando movilizar con éxito recursos humanos y económicos hacia otros países, compartiendo asimismo sus modelos de gestión con diversos actores internacionales (Palma, 2012; Sánchez, et al., 2012).

Entre las experiencias de cooperación más destacadas en el universo de las osc del país, sobresalen las de "ТЕсно", organización que busca superar la situación de pobreza a nivel regional, y de "América Solidaria", organización internacional cuya visión se funda en el desarrollo y la construcción de capacidades en las comunidades y/o personas. En el marco del FCHP, ambas cuentan con valiosas iniciativas de cooperación (Sánchez et al., 2012, ), destacando entre éstas:

a) El Proyecto "Trabajos de invierno 2013 Chile-Paraguay", implementado por TECHO-Chile en conjunto con Agcid, posibilitó a jóvenes voluntarios chilenos y paraguayos combinar esfuerzos para edificar viviendas de emergencia para familias de bajos ingresos de los departamentos de San Pedro y Caaguazú (Paraguay). Dos 
años después, se realizó una nueva versión del proyecto, con el objetivo de integrar y fortalecer el intercambio de saberes entre voluntarios y las comunidades locales. En esta segunda versión participaron 1200 jóvenes chilenos voluntarios de TECHO, y 300 voluntarios paraguayos (Agci-Pnud, 2015; ONU, 2015).

b) El Proyecto "Huertos familiares, comunitarios y educativos para el autoconsumo en Centroamérica” (2013-2017), ejecutado por la Fundación América Solidaria -en alianza con el Fondo de Solidaridad e Inversión Social (Fosis) y el Centro de Innovación de Agricultura Sostenible en Pequeña Escala (Ciaspe) de México, y con financiamiento de Agcid- en Guatemala y Honduras, buscaba ofrecer una alternativa alimenticia de calidad a familias en condición de malnutrición y/o exclusión mediante la implementación de huertos de autoconsumo agroecológicos en 4 comunidades locales. Cabe destacar que, en concordancia con la metodología de América Solidaria, se forjaron alianzas con un socio local de cada país para garantizar la sostenibilidad del mismo (América Solidaria, 2017).

\subsection{Los desafíos de las alianzas estratégicas entre organizaciones de la sociedad civil y el Estado en el marco de la cooperación internacional chilena}

Pese a las múltiples ventajas que conlleva el establecimiento de asociaciones estratégicas para el desarrollo entre las osc y el Estado en el ámbito de la CID, existen igualmente numerosos desafíos que deben ser afrontados para asegurar el éxito y la sostenibilidad de estas alianzas de cooperación. Entre los más importantes, podemos relevar algunos (Sánchez et al., 2012; Guerra, 2014; Ramis, 2018; Moreno, 2019):

- Las osc deben contar con espacios establecidos de participación (seminarios, talleres), que permitan generar un diálogo permanente con Agcid, en donde todos los actores expresen sus inquietudes y sugerencias. Para ello, se debe aprovechar el potencial que la Mesa Multi Actores dispone para construir redes de innovación e intercambiar experiencias, conocimientos y buenas prácticas entre estas entidades y el Estado.

- Existe la carencia de un relato compartido entre el Estado y la sociedad civil, que articule un discurso que legitime socialmente una política más activa en esta materia. Por esto, resulta crucial que se diseñen estrategias que promuevan la generación de una masa crítica de ciudadanos que sean conscientes de las problemáticas regionales, esto considerando que la opinión pública no se encuentra sensibilizada con la cooperación que se otorga a otros países, por lo cual las osc que desarrollan actividades de CID suelen ser criticadas por no otorgar prioridad a los problemas internos del país. Por lo demás, existe un bajo consenso en el país sobre lo que significa ser donante de cooperación y, por lo tanto, sobre cuál es el rol de la cooperación dentro del desarrollo nacional.

- Es necesario que tanto la sociedad civil como el Estado exploren nuevas formas 
de planificación y evaluación de la cooperación en una lógica de colaboración intersectorial, en pos de medir su impacto real. Esto posibilitará replicar proyectos exitosos y buenas prácticas de cooperación.

- La css, al ser una herramienta de la política exterior del país, resulta en ocasiones poco idónea para la acción de las osc, puesto que este ámbito es difícilmente permeable a la participación social.

- Las osc deben lidiar con dificultades de naturaleza institucional al cooperar en el extranjero, como son el desconocimiento de las claves culturales y técnicas específicas que reclama esta labor. Asimismo, poseen un limitado grado de internacionalización, consecuencia de una concentración de las mismas en asuntos domésticos, y de los escasos recursos financieros de que disponen muchas de ellas.

- Desde el punto de vista de las osc, la cooperación gubernamental debe apuntar a gestionar iniciativas más solidarias, y menos asistencialistas, con proyectos que sean sostenibles en el tiempo y que impacten positivamente en las políticas públicas de los países beneficiarios.

- Las osc se han consolidado como críticas al modelo neoliberal, siendo parte de los forjadores de nuevas maneras de entender el desarrollo, lo que ha tensionado sus vínculos con el gobierno.

- Se debe considerar que la horizontalidad de las alianzas multiactor no generan necesariamente más eficacia ni mejores resultados.
- Es imperioso establecer un marco normativo y jurídico que ordene y regule los vínculos entre las Osc y Agcid.

\section{REFLEXIONES FINALES}

Chile se encuentra en un momento de transición en su modelo de cooperación.

Por un lado, su graduación como país de renta alta se vincula con la idea de que los países deben asumir las responsabilidades que comporta su propio desarrollo, cuestión que no buscamos discutir. No obstante, el empleo de límites arbitrarios de ingresos para definir categorías de desarrollo genera nuevas y complejas consecuencias para el país en el ámbito de la CID. La Agenda 2030 además ha aportado nuevos marcos de acción para la CID chilena, motivando la revisión en las formas de proceder en el ámbito de la cooperación nacional. El país debe ser capaz de repensar una cooperación diferente, acorde con los retos y desafíos globales, y con instrumentos y mecanismos innovadores de cooperación.

Para que esto ocurra, será crucial promover la incorporación de nuevos actores a los esfuerzos de cooperación que realiza el país. Este aspecto constituye un pendiente que Chile debe subsanar, si consideramos su condición de donante acotado y los nuevos compromisos y responsabilidades que la Ley de Modernización de Cancillería confiere a Agcid, como institución coordinadora del Sistema Nacional de Cooperación Internacional para el Desarrollo, y las nuevas atribuciones otorgadas en materia de ayuda humanitaria.

Las OsC -y de manera particular las ONGfueron destinatarias preferentes de la coopera- 
ción internacional durante la dictadura militar, ejerciendo un rol de oposición a este régimen autoritario. No obstante, luego del retorno de la democracia, estas organizaciones se vieron impelidas a buscar formas de auto gestionar recursos para poder sobrevivir, logrando algunas de ellas desplegar una importante presencia regional e internacional mediante la conformación de redes de cooperación trasfronterizas.

El conjunto de las osc chilenas posee un importante capital de recursos y capacidades, consolidándose en años recientes como actores aventajados para el desarrollo por su adaptabilidad, flexibilidad y creatividad para diseñar soluciones, su potencial como canalizadoras de recursos y su inestimable legitimidad social, por nombrar algunas. De este modo, pueden aportar a generar una visión holística, integradora y participativa en el marco del establecimiento de alianzas estratégicas multi actorales de cooperación.

Empero, no existe aún desde Agcid un consenso en cuanto a las expectativas reales sobre la participación de este sector. Esto ha derivado en una baja vinculación entre el Estado y las osc en el ámbito de la cooperación.

Como desafío futuro, Agcid debe establecer mecanismos normativos claros para una efectiva participación de las osc en el sistema de cooperación internacional para el desarrollo chileno y, a la vez, generar espacios reales de encuentro en los que estas organizaciones, y otros actores del desarrollo nacional, puedan contribuir con sus capacidades, sus recursos y su expertise. Esto considerando que la cooperación solo es efectiva si en ella participan activamente los actores de la sociedad civil.

\section{REFERENCIAS}

Agci. (2010). Balance Agencia de Cooperación Internacional de Chile período 2006-2010. Recuperado dehttps://www.agci.cl/images/centro_documentacion/bgi/balance_agci_2006_2010.pdf

Agci-Pnud. (2015). Fondo Chile contra el Hambre y la Pobreza. Recuperado de https://www.undp.org/ content/dam/chile/docs/fondo-chile/undp_cl_ fondochile-Brochure-2015.pdf

Agci. (2015). Estrategia Chilena de Cooperación Internacional para el Desarrollo. Recuperado de https://www.agci.cl/images/centro_documentacion/3_ESTRATEGIA_DE_COOPERACION_VersionFinal.pdf

Agcid. (2018). Apuntes desde el Sur: desarrollo, cooperación internacional y política exterior. Recuperado dehttps://www.agci.cl/images/centro_documentacion/Apuntes_desde_el_Sur_2018.pdf

Agcid. (2019). Informe Cuenta Pública 2018. Recuperado de https://www.agci.cl/cpublica/docs/ cuenta_publica_2018.pdf

Agcid-Pnud. (2019). Iniciativas de Cooperación del Fondo Chile contribuyen al desarrollo de otras naciones. Segunda fase 2015-2020. Recuperado de https://www.undp.org/content/dam/chile/ docs/fondo-chile/undp_cl_fchp2020.pdf

Alemany, C. y Freres, C. (2018). América Latina en busca de una inserción efectiva en la agenda internacional de la cooperación para el desarrollo. Revista Internacional de Cooperación y Desarrollo, 5 (1): 59-84.

América Solidaria. (2017). Sistematización huertos familiares, comunitarios y educativos para el autoconsumo en Centroamérica. Recuperado de http://chile.americasolidaria.org/wp-content/ uploads/2017/11/Sistematizacio\%cc\%81nhuertos.pdf 
Amexcid. (2019). Estudio comparativo de 16 agencias de cooperación internacional para el desarrollo: Insumos para su análisis y reflexiones. Recuperado de https://www.gob.mx/cms/uploads/attachment/ file/474927/Estudio_Compartivo_Agencias_de_ Cooperaci_n-comprimido.pdf

Ampuero, R. (22/06/2018). La Graduación de Chile. La Tercera. Recuperado de https://www.latercera.com/opinion/noticia/la-graduacion-chile/216260/

Apci. (2016). Las alianzas multiactor de cooperación internacional para el desarrollo sostenible. Recuperado de http://www.apci.gob.pe/Novedades/ Marco\%20Conceptual\%20Alianzas\%20Multiactor31032017.pdf

Ayllón, B. (2010). El impulso a la cooperación Sur - Sur en América Latina: ¿Nuevas formas de cooperación regional? Breviarios de Relaciones Internacionales (16): 1-12

Ayllón, B. (2012). La cooperación Sur-Sur en América Latina. En Cairou et al., Actas Congreso Internacional América Latina: La autonomía de una región. Madrid, España: 1253-1269.

Ayllón, B. (27-28/08/2012). Cooperación Sur-Sur, organizaciones de la sociedad civil y desarrollo de capacidades en América Latina. Retosy Nuevos Temas en la Relación entre Gobiernos y Sociedades Civiles. Seminario llevado a cabo en Ciudad de México.

Ayllón, B. (2014). La gobernanza de la cooperación y el rol de las organizaciones de la sociedad civil en la cooperación Sur-Sur. Revista Internacional de Cooperación y Desarrollo, 1 (2): 57-89.

Ayllón, B. (2015a). La cooperación Sur-Sur en América Latina y el Caribe. De una época dorada a una fase incierta. Anuario de Integración (11): 134-170.

Ayllón, B. (2015b). Los desafíos de la participación de las organizaciones de la sociedad civil en la coo- peración Sur-Sur. Documento de Trabajo del CIES (10): 1-14.

Calvento, M. y Rolandi, M. (2015). Reconfiguración de la cooperación Sur-Sur en la región latinoamericana y la participación internacional de los actores subnacionales. Revista Mexicana de Ciencias Politicas y Sociales, 15 (224): 315-348.

Cepal. (2015). Financiamiento para el desarrollo en América Latina y el Caribe. Un análisis estratégico desde la perspectiva de los países de renta media. Recuperado de https://repositorio.cepal.org/bitstream/handle/11362/37767/1/S1500127_es.pdf

Cepal. (2018). Nuevos desafíos y paradigmas: perspectivas sobre la cooperación internacional para el desarrollo en transición. Recuperado de https://repositorio.cepal.org/bitstream/handle/11362/44121/1/S1800936_es.pdf

Delamaza, G. (2009). onG, sociedad civil y democracia en Chile post autoritario. En Usos y Abusos de la Sociedad Civil. Plataforma Democrática en Buenos Aires, Buenos Aires.

Díaz de Valdés, L. (2016). Trayectorias en cambio: Un recorrido por la historia de las organizaciones de la sociedad civil. Centro de Políticas Públicas UC. Recuperado de https://politicaspublicas.uc.cl/wpcontent/uploads/2016/11/Cuartoinforme-3.pdf

Egaña, R. (1991). La cooperación internacional: el caso de Chile. En Centro de Documentación, Agci. Recuperado de http://www.agci.cl/attachments/ article/636/coop_int_caso_chile.pd

Fernández, M. (2018). Retrospectiva de la sociedad civil organizada en Chile: 25 años de Acción. Recuperado de http://accionag.cl/wp-content/ uploads/2018/12/Retrospectiva-de-la-sociedad-civil-organziada.-25-a\%C3\%B1 os-deACCI\%C3\%93N..pdf

Ffrench Davis, R. (2016). Chile y la financiación innovadora para el desarrollo: una experiencia para hacer 
globalización inclusiva. Estudios Internacionales (183): 67-92

González, V. (2018). La participación de las OSC en el contexto de la Alianza Global para la Cooperación Eficaz al Desarrollo; el caso de las OSC en México (Tesis de magíster). México D.F.: Instituto Mora.

Granguillhome, R. (2013). La sociedad civil y la cooperación internacional para el desarrollo. Revista Mexicana de Politica Exterior (98): 111-125.

Gruninger, S. (2003). Las ONG durante la transición chilena: Un análisis de su respuesta ideológica frente a su incorporación en políticas sociales de índole neoliberal. Revista MAD (9): 77-181.

Guerra, M. (2014). Agci: análisis de la cooperación para el comercio en el marco de la colaboración Sur-Sur como instrumento de política exterior otorgada en América Latina, durante el período 2006-2013 (Tesis de magíster). Santiago de Chile: Universidad de Chile.

Gutiérrez et al. (2018). Punto de inflexión: Así funciona la cooperación Sur-Sur de las organizaciones de la sociedad civil en Iberoamérica. Actua. Recuperado de file://C:/Users/Catalina/Downloads/PunTO_DE_INFLEXION._Cooperacion_Intelige.pdf

Hernández, R. (2014). La investigación cualitativa a través de entrevistas: su análisis mediante la teoría fundamentada. Cuestiones Pedagógicas (23): 187-210.

Hernández, B. y Lebret, P. (2019). Dos décadas de cooperación europea en Chile: una asociación para el desarrollo. Estudios Internacionales. 51 (192): 35-56.

Huitrón-Morales, A. (2016). La cooperación Sur-Sur y el reto de su cuantificación, evaluación y valoración. Revista Iberoamericana de Estudios de Desarrollo. 5(1): 88-122.

Irarrázaval, I. et al. (2016). Mapa de las organizaciones de la sociedad civil 2015. Pontificia Universidad Ca- tólica, Centro de Políticas Públicas uc. Disponible en https://politicaspublicas.uc.cl/wp-content// uploads/2016/01/PDF-Brochure-Mapa-de-lasOrganizaciones.pdf

Lazo, M. (2012). Chile y la cooperación internacional: 20 años de experiencia. En Artaza, M. y Ross, C. (coord.), La politica exterior de Chile 1990-2009: del aislamiento a la integración global, Santiago de Chile: RIL Editores, 367-396.

López, D. (2015). Las estrategias de desarrollo económico del sector público en Chile: El caso de la exportación de servicios a partir de 1990 (Tesis de doctorado). Santiago: Universidad de Chile.

Ministerio Secretaría General de Gobierno. (2016). Guía Fondos de Cooperación Internacional 2015. Recuperado de http://participemos.gob.cl/wpcontent/uploads/2016/03/fondos-internacionales.pdf

Moreno, L. (24/05/2019). ¿Por qué una cooperación entre Múltiples Actores? Un bienestar compartido requiere de la academia, la sociedad civil y el sector privado. Presentado en Taller "El rol de la sociedad civil". Santiago de Chile: Agcid.

ONU. (31/07/2015). 1.200 jóvenes voluntarios chilenos construirán viviendas en Paraguay con el apoyo del Fondo Chile. Recuperado de http://www. onu.cl/es/1-200-jovenes-voluntarios-chilenosconstruiran-viviendas-en-paraguay-con-el-apoyodel-fondo-chile-3/

Palma, A. (2012). La cooperación y los nuevos donantes: La experiencia de Chile. Economistas sin Fronteras, $N^{\circ}$ 5. Recuperado de https://fae.usach.cl/fae/ docs/opinion/cooperaci\%C3\%B3n_y_los_nuevos_donantes.pdf

Ramis, A. (2018). Contexto político y regulatorio de la cooperación internacional chilena. Una mirada desde la sociedad civil. Acción-Aoed. Recuperado de http://accionag.cl/wp-content/ 
uploads/2018/05/Contexto-pol\%C3\%ADtico-yregulatorio-de-la-cooperaci\% $3 \%$ B3n-internacional-chilena.-Una-mirada-desde-la-SociedadCivi-1.pdf

Rodríguez, G. et al. (1996). Metodología de la investigación cualitativa, Granada, España: Ediciones Aljibe.

Rodríguez, L. (2017). El desarrollo de las ONG de México y su coincidencia con los Objetivos para el Desarrollo Sostenible de Naciones Unidas. Revista de Economia Pública, Social y Cooperativa (91): 59-84.

Ruiz, L. (2019). 40 años de cooperación Sur-Sur ¿̇iene Iberoamérica algo que enseñar? Análisis Carolina. Recuperado de https://www.fundacioncarolina. es/wp-content/uploads/2019/03/ac3.pdf

Sanahuja, J. (2014). De los Objetivos del Milenio al desarrollo sostenible: Naciones Unidas y las metas globales post-2015. En Mesa, M. (Ed.), Focos de tensión, cambio geopolítico y agenda global (pp. 49-83). Madrid, España: Ceipaz.

Sánchez, F. (2010). Análisis de la actuación de Chile en la cooperación internacional para el desarrollo en sus modalidades Sur-Sur y triangular. Documento de Trabajo $\mathrm{N}^{\circ}$ 18. Madrid: Instituto Universitario de Desarrollo y Cooperación, Universidad Complutense de Madrid.

Sánchez, A. et al. (2012). Una reflexión desde el sector público, la academia y la sociedad civil chilena. Recuperado de https://www.agci.cl/images/newsletter/tensiones_oportunidades_chile.pdf
Santander, G. (2018). Chile como oferente de cooperación Sur-Sur: la progresiva adaptación a los nuevos tiempos y responsabilidades. Estudios Internacionales, 50 (190): 9-35.

Soto, F. y Viveros, F. (2016). Organizaciones de la sociedad civil en Chile: propuestas para financiamiento público y fortalecimiento institucional. Revista Latinoamericana, 15 (45): 429-454.

Surasky, J. (6-8/09/2017). Cooperación Sur-Sur en la Agenda 2030 y hacia el Paba+40. Presentado en la Sesión de Alto Nivel del Foro de Cooperación al Desarrollo de 2017. Buenos Aires: Ecosoc.

Van Klaveren, A. (2011). La política exterior de Chile durante los gobiernos de la Concertación. Estudios Internacionales (169): 155-172.

Weber, G. (2011). De la Declaración de París hacia el Iv Foro de Alto Nivel en Busán. Perspectivas desde Ecuador y de la región América Latina y el Caribe. En Weber, G. (Coord.), Debates sobre cooperacióny modelos de desarrollo. Perspectivas desde la Sociedad Civil en el Ecuador (pp. 21-46). Quito: Ciudad.

Zamora, E. y Molina, C. (2016). Las organizaciones de la sociedad civil y los principios y alternativas regionales de la cooperación Sur-Sur. En Ayllón, B. (Coord.), ¿Latinoamérica dividida? Procesos de integración y cooperación Sur-Sur (pp. 177-198). Quito: Editorial IAEN. 